\title{
Pilot-Assisted 16-Level QAM for Wireless Video
}

\author{
Hamid Gharavi, Fellow, IEEE
}

\begin{abstract}
This paper presents a twin-class transmission system for narrowband radio access channels suitable for handheld video phone and multimedia portable PC applications. The transmission system is comprised of a hierarchical 16-QAM modulation technique and a channel-coding scheme. The formation of dual-priority transmission is due to differing error resiliencies of the bits that make up a given symbol in a Gray-coded 16-QAM. On this basis, a twin-class pilot-assisted fade-estimation technique that can gracefully reduce the power loss caused by the transmission of pilot overhead is developed. The twin-class 16-QAM system is then used to transport a compressed video bitstream, which is partitioned to match the bit-error sensitivity of the transmitted symbol. The partitioning scheme is based on a separation of the variable-length (VL) coded discrete cosine transform (DCT) coefficients within each DCT block. This partitioning scheme is then applied to split the ITU-T H.263-coded bitstream. The scheme is suitable for constant bit-rate transmission (CBR), where the fraction of bits assigned to each of the two partitions can be adjusted according to the requirements of the unequal error protection scheme employed. The distribution of the VL-coded (VLC) information amongst the two partitions is performed adaptively. Finally, the performance of the partitioning scheme for transmission of video signals using our twin-class 16-QAM transmission system is evaluated under multipath fading conditions.
\end{abstract}

Index Terms-QAM modulations, unequal error protection, video partitioning, wireless video, 16-QAM.

\section{INTRODUCTION}

$\mathbf{T}$ HE DESIGN of multimedia terminals was conceived with the assumption that users do not move and the transmission medium is almost error free. As mobility is becoming a major challenge in future multimedia communications, an essential issue is how to protect a highly sensitive video portion of multimedia information against hostile multipath fading environments. Since most existing video compression standards have been developed for relatively benign, near-error-free environments, they cannot be directly applied in a hostile mobile domain. This is mainly due to the extensive employment of variable-length coding (VLC) techniques, which are efficient in bit-rate reduction terms, but are error sensitive, since a single transmission error may result in an undecodable string of bits. In addition, to enhance the compression efficiency, interframe coding is normally deployed. However, the main drawback with interframe coding for mobile applications is the transmission

Manuscript received January 20, 1998; revised December 14, 2001. This work was supported in part by the Defence Advanced Research Projects Agency under Contract AO L738. This paper was recommended by Associate Editor J. Brailean.

The author is with the National Institute of Standards and Technology (NIST), U.S. Department of Commerce, Gaithersburg, MD 20899-8920 USA (e-mail gharavi@antd.nist.gov).

Publisher Item Identifier S 1051-8215(02)02017-7. of error bursts which tend to propagate to the proceeding interframe-coded video signal. To avoid this, less error-prone intraframe methods which lack coding efficiency should be considered. An alternative approach which would allow the utilization of interframe coding is transmission via separate channels using an unequal error-protection strategy. For instance, it is shown in [1] and [2] that the so-called maximum-minimum distance Gray-coded 16-QAM phasor constellation naturally forms two different-integrity subchannels. Specifically, the higher integrity 16-QAM subchannel-referred to as the C-1 subchannel-is represented by the most significant bit (MSB) of both the in-phase $(I)$ and quadrature-phase $(Q)$ bits. By contrast, the lower priority subchannel $(\mathrm{C}-2)$ is formed by the remaining 2 bits of the 4-bit 16-QAM symbol, i.e., least significant bits (LSBs). The associated bit-error-rate (BER) difference of the $\mathrm{C}-1$ and $\mathrm{C}-2$ subchannels depends on the transmission channel condition, which is Rayleigh in the worst case, when no line-of-sight (LOS) path is present between the transmitter and receiver. When there is a LOS path, various Ricean channels are encountered.

In QAM-based video transceivers, it is possible to exploit the BER differences of the $\mathrm{C}-1$ and $\mathrm{C}-2$ subchannels in providing video bit sensitivity-matched error protection. This BER difference can be further augmented, if required, by the different video bit sensitivities or equalized by employing different channel codecs. Alternatively, a range of different channel codecs can be considered in order to create a more finely graded set of bit-protection classes. We note, furthermore, that in 64and 256-QAM, there are three and four different protection classes, respectively.

In this paper, we first present a detailed analysis of the $\mathrm{C}-1$ and C-2 bit-error probabilities for Rayleigh fading channels. In addition, to compensate the effects of the channel-induced magnitude- and phase-fluctuations pilot-assisted modulation (PSAM), a technique first proposed by Cavers [3], is investigated. Please note that the PSAM technique inserts known pilot symbols in the transmission bursts, which essentially sample the channel's complex fading envelope according to the constraints of Nyquist's sampling theory. To take advantage of the BER difference of the C-1 and C-2 subchannels, a twin-class pilot assisted fade estimation technique that can gracefully reduce the power loss caused by the pilot overhead is presented. A combination of twin-class PSAM and channel coding is then deployed to asses the overall performance of the Gray-coded 16-QAM. The transmission system is then conferred for transporting video-coded bitstreams over multipath fading channels. Thus, the second part of this paper deals with bitstream splitting of video signals into two layers for transportation via $\mathrm{C}-1$ and $\mathrm{C}-2$ channels. This part includes the application of the bitstream partitioning to the ITU-T H.263 


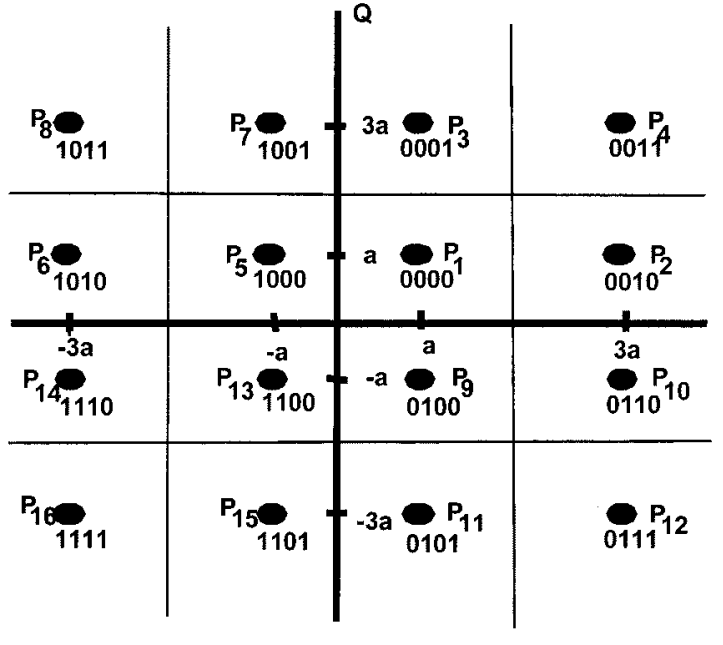

Fig. 1. Gray-coded 16-QAM constellation.

coding standard and the quality assessment of the decoded video after transmission over Rayleigh fading channels using the twin-class 16-QAM system.

\section{GRAY-CODED 16-QAM}

A 16-QAM square constellation is shown in Fig. 1. As can be seen, the distance of a constellation point from the boundary of its decision region (referred to here as the protection distance) for the two MSBs is either "a" or " $\mathbf{3 a}$ " and assuming that the probability of occurrence for all 16 phasors is equal, the BER probability classified here as channel $1(\mathrm{C}-1)$ [1], [2] is given by

$$
P_{\mathrm{C}-1}=\frac{1}{2} Q\left\{\frac{3 a}{\sqrt{N_{0} / 2}}\right\}+\frac{1}{2} Q\left\{\frac{a}{\sqrt{N_{0} / 2}}\right\}
$$

where $Q\{\cdot\}$ is the area under the Gaussian tail and $N$ is the onesided noise spectral density of additive white Gaussian noise (AWGN).

For the two least significant bits (LSB) signifying channel 2 (C-2), the decision distance is " $\mathrm{a}$ " at all times and, thus, the BER probability can be expressed as

$$
P_{\mathrm{C}-2}=Q\left\{\frac{a}{\sqrt{N / 2}}\right\} \text {. }
$$

The average signal energy per symbol $(E s)$ for 16-QAM is

$$
E s=10 a^{2} \text { or } a=\sqrt{\frac{E s}{10}} .
$$

Substituting " $a$ " from the above in (1) and (2)

$$
\begin{aligned}
& P_{\mathrm{C}-1}=\frac{1}{2} Q\left\{\sqrt{\frac{9 \gamma_{s}}{5}}\right\}+\frac{1}{2} Q\left\{\sqrt{\frac{\gamma_{s}}{5}}\right\} \\
& P_{\mathrm{C}-2}=Q\left\{\sqrt{\frac{\gamma_{s}}{5}}\right\}
\end{aligned}
$$

in which

$$
\gamma_{s}=\frac{E_{s}}{N_{0}}
$$

The total probability of error for 16-QAM is

$$
\overline{P_{e}}=\frac{1}{2}\left(P_{\mathrm{C}-1}+P_{\mathrm{C}-2}\right) .
$$

Equations (3) and (4) represent the bit-error probabilities for AWGN channels (nonfading case).

\section{Multipath FADING CHANNELS}

In mobile radio channels the fading signal can be expressed as

$$
S(t)=c(t) \cdot z(t)+n(t)
$$

where $z(t)$ is the modulated signal, $n(t)$ is the AWGN, and $c(t)$ is the multipath gain at the output of the fading channel. For narrowband channels where the signal bandwidth is much smaller than the coherence bandwidth, the received signal undergoes flat fading [5]. Under flat fading conditions, the spectral characteristics of the signal are preserved. Thus, $c(t)$ can be presented as

$$
c(t)=r(t) e^{-j(t)}
$$

where $\varphi(\mathbf{t})$ is the phase shift and $\mathbf{r}(\mathbf{t})$ represents the multiplicative variation in the signal envelope. For slow flat fading channels, the phase shift and amplitude attenuation of the received signal can be assumed to be constant within at least one symbol period. $\varphi$ can be accurately estimated in the detection process (e.g., coherent) and $\mathbf{r}$ is considered to be a Rayleigh-distributed random variable where its probability density function be shown as

$$
p(r)=\frac{r}{\sigma^{2}} e^{\left(-r^{2}\right) / 2 \sigma^{2}}, \quad r \geq 0
$$

where $\sigma$ and $\sigma^{2}$ are the rms voltage and averaged power of the received signal (before envelope detection), respectively. For the slow flat fading model, the signal amplitude during each symbol period is multiplied by a Rayleigh fading factor [7]. Thus, the instantaneous SNR is defined as

$$
\gamma_{i}=r^{2} \frac{E_{s}}{N_{0}}
$$

As indicated in (9), $r$ has a Rayleigh distribution. Consequently, $r^{2}$ has a Chi-square distribution with two degrees of freedom. Thus

$$
p_{e}\left(\gamma_{i}\right)=\frac{1}{\gamma_{0}} e^{-\left(\gamma_{i} / \gamma_{0}\right)}
$$

$\gamma_{0}$ is the average value of the SNR, i.e.,

$$
\gamma_{0}=\overline{r^{2}} \frac{E_{s}}{N_{0}}
$$

where $\overline{\mathbf{r}^{\mathbf{2}}}=E\left\{r^{2}\right\}=2 \sigma^{2}$. Thus, the average BER can be shown as

$$
\overline{p_{e}}=\int_{0}^{\infty} p_{e}\left(\gamma_{i}\right) p\left(\gamma_{i}\right) d \gamma_{i} .
$$


From (3), (11), and (13), the average BER for the first channel (assuming coherent detection) can be shown as

$\overline{p_{\mathrm{c}-1}}=\frac{1}{2} \int_{0}^{\infty} \frac{1}{\gamma_{0}} e^{-\left(\gamma_{i} / \gamma_{0}\right)}\left[Q\left\{\sqrt{\frac{\gamma_{i}}{5}}\right\}+Q\left\{\sqrt{\frac{9 \gamma_{i}}{5}}\right\}\right] d \gamma_{i}$.

Integration by part and after simplification will give us

$$
\overline{P_{\mathrm{c}-1}}=\frac{1}{4}\left(2-\sqrt{\frac{\gamma_{0}}{10+\gamma_{0}}}-\sqrt{\frac{9 \gamma_{0}}{10+9 \gamma_{0}}}\right) .
$$

For the second channel, however, the situation is not as straightforward as for the first channel. The total average error probability of the second channel is shown as

$$
\begin{aligned}
\overline{P_{\mathrm{c}-2}}= & \frac{1}{4} e^{\frac{-\pi \gamma_{0}}{10}}\left[1+\sqrt{\left.\frac{\gamma_{0}}{10+\gamma_{0}} e^{\frac{\pi \gamma_{0}^{2}}{10\left(\gamma_{0}+10\right)}}\right]}\right. \\
& +\frac{1}{4} e^{\frac{-9 \pi \gamma_{0}}{40}}\left[1+\sqrt{\frac{4 \gamma_{0}}{4 \gamma_{0}+10}} e^{\frac{9 \pi \gamma_{0}^{2}}{40 \gamma_{0}+100}}\right] .
\end{aligned}
$$

but to avoid excessive analytical details, its derivation is presented in the Appendix.

Analytical evaluation of the BER performance, using (15) for the first channel and (16) for the second channel, indicates that the second channel has an unacceptable error rate in fading [1]. Indeed, such behavior backed by our simulation confirms that the BER performance of the second channel remains almost unaffected by the average SNR, whereas the BER of the first channel decreases as the SNR increases. In the absence of the multipath effect (i.e., a nonfading environment), the difference between the two channels seems to be marginal. The poor BER performance of the second channel indicates that the bandwidth efficiency of multilevel modulation cannot be adequately utilized due to envelope fluctuations which tend to drastically affect the detection of the two least significant bits. To overcome such a deficiency, we have developed a PSAM scheme which takes advantage of the twin-class property of the 16-QAM modulation system.

\section{FADE ESTIMATION AND COMPENSATION}

In conventional pilot-symbol-assisted modulation [3], [4], a symbol representing a known phasor is allocated at the beginning of each transmitting frame consisting of $K-1$ symbol data. For a symbol period of $T_{s}$, the frame length corresponds to

$$
T_{f}=K \cdot T_{s}
$$

The sample timing of each symbol in the $n$th frame $\left(t_{n, i}\right)$ can be shown as

$$
t_{n, i}=n \cdot T_{f}+\left(\frac{i}{K}\right) T_{f}, \quad i=0,1,2, \ldots K-1 .
$$

If a symbol representing a known phasor $\mathbf{P}_{\mathbf{A}}$ (e.g., $A=16$ in Fig. 1) is transmitted at the beginning of a frame, the demodulated pilot symbol at each frame timing (i.e., $t_{n, 0}=n \cdot T_{f}$ ) is given by

$$
R\left(t_{n}, 0\right)=C\left(t_{n}, 0\right) P_{A}+n\left(t_{n}, 0\right)
$$

At the demodulator, the ratio of the received signal over the known phasor (i.e., $R\left(t_{n, 0}\right) / P A$ ) represents the estimated value of $C\left(t_{n, 0}\right)$ where

$$
\bar{C}\left(t_{n}, 0\right)=C\left(t_{n}, 0\right) P_{A}+\frac{n\left(t_{n}, 0\right)}{P_{A}} .
$$

The above equation indicates that the estimate of the pilot symbol is the sample value of $C\left(t_{n, 0}\right)$ affected by noise. The estimated symbols for consecutive frames can then be used to interpolate the symbol-spaced samples of the received signal $R\left(t_{n, i}, 0\right)$. Optimal interpolation using a Gaussian or Wiener filter can be used but, to avoid the computational complexity of the latter, the former has been considered here.

The next step in pilot-symbol-assisted fade estimation is choosing a suitable frame length. Bear in mind that a large value for $K$ can reduce the interpolation accuracy, thus leading to a higher BER. At the same time, an unnecessarily small value for $K$ can reduce the QAM spectral efficiency due to power loss. For instance, for a frame length of $K$ symbols of a source coded at a bit rate of $R_{b}$ bits/s, an increase in overhead would be $R_{b} / K$ bits/s. However, an optimum value for the pilot spacing can be found in accordance with the fading sampling rate. For a given transmission system, the only changing parameter affecting the fading rate is the velocity of the mobile unit, which translates to the Doppler Spreading, defined as

$$
f_{D}=\left(v \cdot f_{c}\right) / c
$$

where

$f_{D}$ maximum Doppler frequency;

$v \quad$ velocity of the mobile unit;

$f_{c} \quad$ carrier frequency;

c speed of light.

In our experiments, the Doppler shift is normalized by the transmitted symbol rate (i.e., $f_{D} T_{s}$ ). For our Rayleigh fading simulated model, a flat fading model with the power spectrum suggested by Jakes [6] has been used. The carrier frequency was set at $1.9 \mathrm{GHz}$, with a baud rate of 24000 . The over-sampling rate was 8 and the pulse shaping roll factor was 2.5. Fig. 2 shows the effect of frame size on the BER performance of both channels using second order interpolation. In this figure, two normalized Doppler shifts, $f_{D} T_{s}=0.01$ and $f_{D} T_{s}=0.025$, were considered.

The results indicate that while the first channel can tolerate a much larger frame size, the second channel would require a more frequent pilot update as its BER performance tends to deteriorate more rapidly. Based on this observation, we present a hierarchical PSAM scheme which allows more frequent pilot transmission for the second channel. In addition, by employing a half-symbol pilot, a substantial increase in the overhead can be avoided. The proposed strategy is depicted in Fig. 3, which shows how the pilot symbol is split into two halves, where each is transmitted at a different frame rate. As shown in this figure, the pilot symbol $P_{A}$ is formed by combining the two half-symbols (i.e., $P_{\mathrm{C}-1}=$ "11," $P_{\mathrm{C}-2}=$ "11" which correspond to $P_{16}=$ "1111" in Fig. 1). The full symbol formation can occur at a multiple frame length (i.e., super-frame) $T_{\text {sup }}=j \cdot T_{f}$. For the second channel, a half-symbol (i.e., $P_{\mathrm{C}-2}=$ " 11 ") is inserted at 


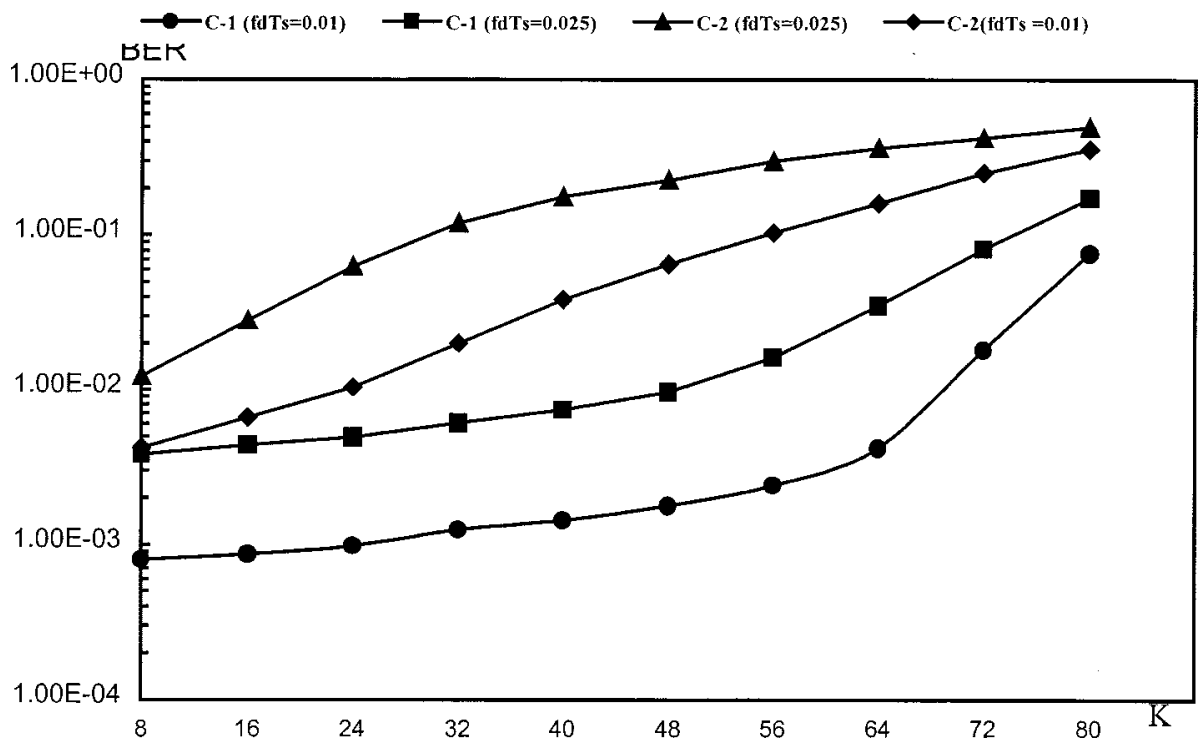

Fig. 2. Effect of frame size on the BER performance of C-1 and C-2 channels for SNR $=30 \mathrm{~dB}$.

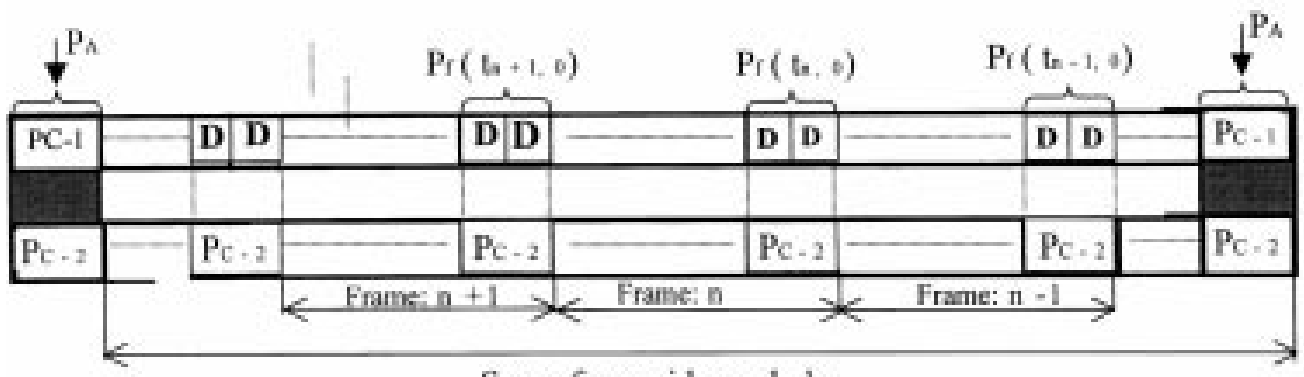

Super frame: $\mathrm{j} . \mathrm{k}$ symbols

Fig. 3. Framing structure for transmission of pilot information.

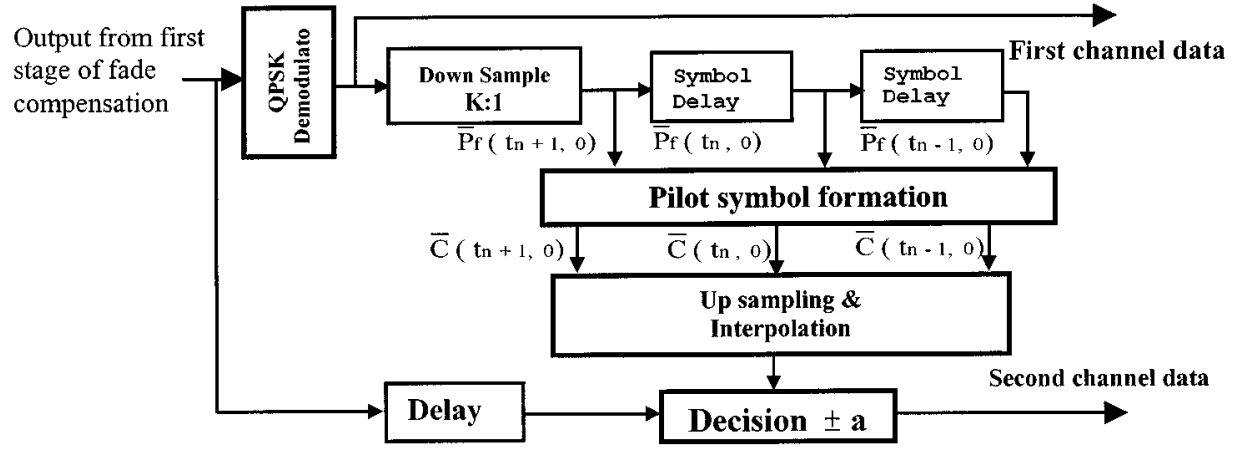

Fig. 4. Secondary fade estimation and compensation for the second channel data.

the beginning of each frame $T_{f}$. The half symbol $P_{\mathrm{C}-2}=$ " 11 " corresponds to a phasor repressing the maximum amplitude, but its exact location (phase) in the 16-QAM constellation is determined by its corresponding two bits which are transported via the first channel (i.e., $P_{f}(\cdot, \cdot)$ in Fig. 1).

In this arrangement, the $P_{A}$ pilot (first channel; $P_{\mathrm{C}-1}=$ " 11 ") is primarily used to estimate the channel for the recovery of the first channel data (primary channel estimation). Upon detection of the first channel data at a super frame basis, the second stage of interpolation is performed on a shorter frame (i.e., $\mathbf{T}_{\mathbf{f}}$ ) to recover the second channel data. Fig. 4 shows the configuration of the fade estimation and compensation scheme for the twochannel 16-QAM system.

In this configuration, after a primary-stage of fading estimation and compensation at a super-frame basis, the demodulated signal is first split into two branches. For the first branch, the decision boundaries for both the $I$ and $Q$ components are set at zero-level (i.e., using a QPSK demodulator). The first two bits of the decoded first channel data at the beginning of each frame, together with the $P_{\mathrm{C}-2}$ pilot bits (e.g., $P_{\mathrm{C}-2}=$ "11"), form a symbol estimate of the transmitted phasor. As shown in Figs. 3 and 4 , the estimated fade symbols of three consecutive frames 


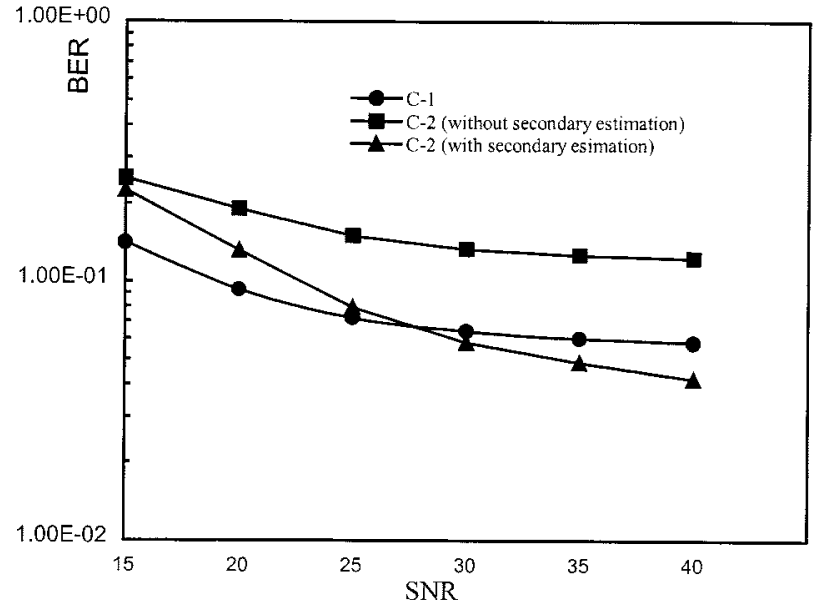

(a)

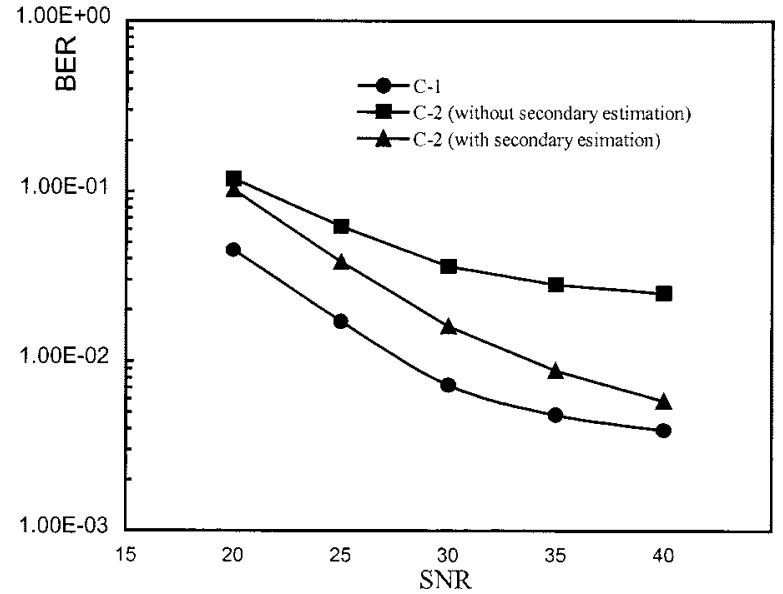

(b)

Fig. 5. Effect of secondary fade compensation on the BER performance of the second channel data. (a) $f_{D} T_{s}=0.015$. (b) $f_{D} T_{s}=0.01$.

are subsequently used to perform symbol-spaced interpolation according to (20). This is then applied to scale and rotate the signal constellation in accordance with the decision boundaries for the $I$ and $Q$ components for recovering the second channel data.

The above scheme was implemented using Signal Processing Work System (SPW) simulation tools. ${ }^{1}$ The simulation model was then evaluated by using a frame of 8 symbols $(k=8)$ and a super-frame of 64 symbols (e.g., $j=8$ ). Fig. 5 shows the BER results for channel SNR ranging from 15 to $40 \mathrm{~dB}$ for $f_{D} T_{s}=0.015$ [Fig. 5(a)] and $f_{D} T_{s}=0.01$ [Fig. 5(b)]. From these results, it can be clearly observed that the performance of the second channel is considerably enhanced, particularly at higher channel SNRs. This is due to the fact that at a low channel SNR, the error probability of the first channel data increases, which could consequently affect the accuracy of pilot symbols used for secondary fade estimation. However, we should point out that due to the hierarchical structure of the system (e.g., partitioned input video data), once the first channel data frame is

${ }^{1}$ SPW is a registered trademark of Cadence Design Systems, Inc. The SPW is identified in this paper to foster understanding. Such identification does no imply recommendation or endorsement by the National Institute of Standards and Technology, nor does it imply that this product is necessarily the best available for the purpose.

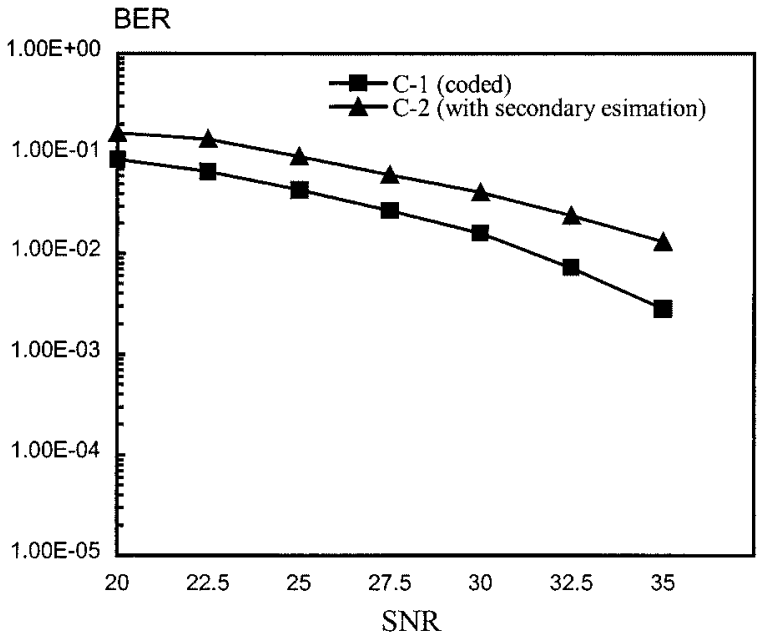

(a)

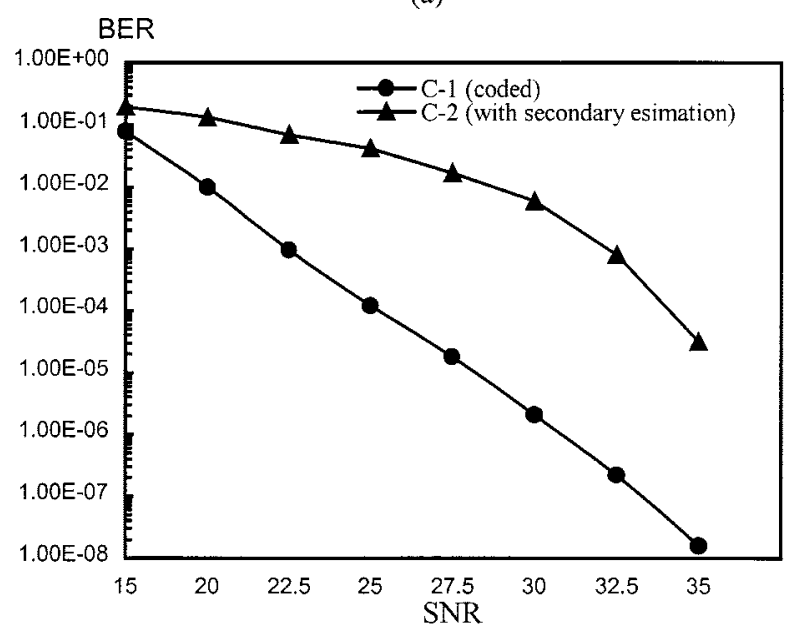

(b)

Fig. 6. BER performance of the coded first channel and its effect on the uncoded second channels. (a) $f_{D} T_{s}=0.02$. (b) $f_{D} T_{s}=0.01$

corrupted by errors, the data transmitted on the second channel would no longer be of any use. Thus, improving the performance of the first channel (e.g., by means of forward error correction (FEC) coding) can consequently enhance the performance of the second channel.

Block coding such as Reed-Solomon (RS) and BCH (outer coding) combined with convolutional coding (inner coding) can provide a powerful error protection tool against multipath fading for mobile applications. In this paper, however, we have considered convolutional coding for the first channel followed by a block interleaver to disperse the error bursts. At the receiver, a hard decision Viterbi decoder is then applied (after de-interleaving) to recover the first channel data. The decoded firstchannel data is subsequently used to help the channel estimation for the second channel. Fig. 6 illustrates the BER performance of the first and second channels using a 1/2 rate convolutional code with a constraint length of 9 for both $f_{D} T_{S}=0.02$ [Fig. 6(a)] and $f_{D} T_{S}=001$ [Fig. 6(b)]. As indicated in these figures, at higher SNR values where the FEC on the first channel becomes more effective, the BER performance of the second channel improves considerably. This behavior appears to fit very well if the input video bitstream is partitioned in such a way that 
the first channel can carry the most error-sensitive information of the compressed video. Such a strategy will be discussed next.

\section{VIDEO PARTITIONING}

In this section, we are mainly concerned with developing partitioning schemes suitable for transmission of compressed video over our twin-class 16-QAM system. The objective is to exploit the BER differences of the $\mathrm{C}-1$ and $\mathrm{C}-2$ subchannels by providing video bit sensitivity-matched error protection. Since the transmission rate for $\mathrm{C}-1$ and $\mathrm{C}-2$ has to be at fixed rates with equally sized partitioning, we have devoted our attention to constant bit-rate (CBR) transmission. Since C-1 and C-2 use different PSAMs and error-protection overheads, the partitioning algorithm should be able to divide the original bitstream into any desirable sized partitions. The partitioning process is, in general, described with the aid of the fraction of bits assigned to the individual partitions. The number of different bit-sensitivity classes and the video bits assigned to them have to be decided on the basis of the visual importance differences of the various video bits.

In this paper, our main objective has been to develop an efficient partitioning scheme to split an already-compressed video into two bitstreams for the transmission of video signals over mobile channels. As will be discussed in the following section, the ITU-T H.263 [9] video-coding standard has been considered to compress the video signal before partitioning.

\section{A. Hybrid-Based Video Partitioning}

The so-called inter-frame hybrid DCT coding technique has been adopted for most practical video codecs, including the existing video-coding standards [8]-[12]. The multiplexing structure for transmission of video-coded information of these standards is generally based on the same concept. For example, in the H.263 standard, the video-coded information for each frame is arranged in four hierarchical layers such as a picture layer, group of blocks (GOB) layer, macroblock (MB) layer, and block layer.

The picture layer contains a number of codewords that include the picture start code (PSC), picture type (PTYPE) indicating whether the frame is INTER (P-picture) or INTRA (I-picture) coded, video format, and other important parameters that are used to encoded the frame. The picture layer is then followed by a GOB layer representing a row of contiguous MBs within the coding frame. The GOB header contains information such as GOB start code, group number (GN), and the quantization parameter to be used for the GOB blocks.

Next is the MB layer; its header begins with a single bit, indicating whether any more information is transmitted for the MB (this is referred to as COD). For instance, if the COD flag indicates a coded $\mathrm{MB}$, its header provides information such as the coded block patterns for chrominace (CBPCM) and luminance (CBPY), which identifies the coded blocks within a MB. In addition, the codewords representing the horizontal and vertical components of motion vectors for the MB is included in the header. The final layer of the video syntax is the block layer which contains the VLC-coded information of the DCT coefficients.
As described above, it is essential that the decoder receive the header information without errors to ensure that the image is reconstructed correctly. For instance, if the header information is corrupted during transmission, the decoder will have no indication as to how the frame, GOB, or MB has been coded and so any further data received will be useless. The actual DCT coefficients are transmitted at the block layer and errors detected in this data mean that some, or all, of the data is lost. In this case, if errors affect only higher frequency coefficients, the damage would be less catastrophic as the block can be reconstructed with minimal visual distortion. It is therefore beneficial to partition the coded bitstream in such a way that the header information and possibly as many lower frequency DCT coefficients, are better protected. However, the major challenge in partitioning the video-coded bitstream is to avoid any substantial increase in the bit rate. This, to a large extent, depends on the way in which the visual data is divided and subsequently transmitted.

\section{B. Partitioning of DCT Coefficients}

As discussed above, a video frame is first divided into nonoverlapping blocks $\left(S_{f}\right)$ and then each block is transformed by a DCT, quantized and entropy coded. In this scheme, the difference between the $f$ th input block $S_{f}$ and its corresponding motion-compensated (MC) predicted block $S_{f-1}^{*}$ (i.e., the reference block) is transformed via a DCT. The output of the block, $\operatorname{DCT}\left(S_{f}-S_{f-1}^{*}\right)$, is then quantized, $\left.Q\left\{\operatorname{DCT}\left(S_{f}-S_{f-1}^{*}\right)\right)\right\}$, and VLC coded before being transmitted.

The separation of the quantized DCT coefficients may be arranged either before or after entropy coding. In the former, referred to as fixed-zone partitioning, the same number of lower frequency coefficients (in a zig-zag scanning order) is selected as the upper-zone for transmission over the first channel. In the latter case, the first partition takes up a fixed number of VLC codewords instead. To distinguish between the two splitting methods, the impact a loss of the second partition would have on the reconstruction of the first partition is presented. Neglecting the effect of quantization noise (e.g., $S_{f-1}=S_{f-1}^{*}$ ), the prediction error signal (before VLC coding) may be shown as follows:

$$
\operatorname{DCT}\left(S_{f}-S_{f-1}\right)=\operatorname{DCT}\left(S_{f}\right)-\operatorname{DCT}\left(S_{f-1}\right)
$$

where $S_{f}$ and $S_{f-1}$ represent the current block of picture elements and its corresponding MC predicted block (i.e., reference block), respectively. For partitioning, a fixed number of top VLC codewords may be selected for the first partition for each block of 8-by-8. In this case, the number of coefficients that a single VLC codeword can represent depends on the number of quantized zero coefficients (i.e., a zero run) preceded by a nonzero value (level). It is assumed that the coefficients are read in a zig-zag scanning order (from the top left to bottom right corners). For instance, the first VLC codeword may represent one or more quantized DCT coefficients of the lowest order, depending on the nature of the run-level symbol. Therefore, for a given number of selected top VLC codewords for the first partition, different blocks may result in covering a different number of DCT coefficients. To assist with the analysis, a zone $Z_{x, y}$ 
$(y>x)$ is defined as a region in which the DCT block covers a specific number of DCT coefficients where the coefficients are identified by the zone's subscript indices $x$ and $y$. For instance, $Z_{x, y}$ corresponds to the DCT coefficients: $C_{x}, C_{x+1}, \ldots C_{y}$ (in a zig-zag order).

Therefore, for a given number of VLC codewords selected for the first partition, it may be assumed that for the current coding block, this would correspond to upper zone $Z_{1, m}$. Thus, the prediction error signal for selected VLC codewords may be shown as follows:

first partition

$$
\begin{aligned}
& Z_{1, m}\left\{\operatorname{DCT}\left(S_{f}-S_{f-1}\right)\right\} \\
& \quad=Z_{1, m}\left\{\operatorname{DCT}\left(S_{f}\right)\right\}-Z_{1, m}\left\{\operatorname{DCT}\left(S_{f-1}\right)\right\}
\end{aligned}
$$

second partition

$$
\begin{aligned}
& Z_{m+1,64}\left\{\operatorname{DCT}\left(S_{f}-S_{f-1}\right)\right\} \\
& \quad=Z_{m+1,64}\left\{\operatorname{DCT}\left(S_{f}\right)\right\}-Z_{m+1,64}\left\{\operatorname{DCT}\left(S_{f-1}\right)\right\} .
\end{aligned}
$$

Assume that when the reference block was encoded (based on the same number of VLC codewords), the number of its DCT coefficients for the first partition was represented by a zone that was smaller than that in the current block (i.e., $Z_{1, n}<Z_{1, m}$ ). Thus, the last term in (23) may be divided as

$$
\begin{aligned}
Z_{1, m} & \left\{\operatorname{DCT}\left(S_{f-1}\right)\right\} \\
& =Z_{1, n}\left\{\operatorname{DCT}\left(S_{f-1}\right)\right\}+Z_{(n+1), m}\left\{\operatorname{DCT}\left(S_{f-1}\right)\right\} .
\end{aligned}
$$

It can be easily deduced from (22) that in order to recover the current DCT block, the prediction error signal should be added to the reference block. Therefore, in the absence of any distortion caused by quantization or transmission errors, the VLC codewords for the first partition can be expressed as

$$
\begin{aligned}
& Z_{1, m}\left\{\operatorname{DCT}\left(S_{f}\right)\right\} \\
& \quad=Z_{1, m}\left\{\operatorname{DCT}\left(S_{f}-S_{f-1}\right)\right\}+Z_{1, m}\left\{\operatorname{DCT}\left(S_{f-1}\right)\right\} .
\end{aligned}
$$

Using (24), (25) may be expressed as

$$
\begin{aligned}
Z_{1, m} & \left\{\operatorname{DCT}\left(S_{f}\right)\right\}=Z_{1, m}\left\{\operatorname{DCT}\left(S_{f}-S_{f-1}\right)\right\} \\
& +Z_{1, n}\left\{\operatorname{DCT}\left(S_{f-1}\right)\right\}+Z_{(n+1), m}\left\{\operatorname{DCT}\left(S_{f-1}\right)\right\} .
\end{aligned}
$$

In the case of transmission errors, it is important to note that the coefficients within the $Z_{n+1, m}$ belong to the second partition at the time the reference block was transmitted.

Next, the situation when this partition $\left(Z_{n+1, m}\right)$ is received erroneously at the time the reference block was transmitted will be considered (this is a realistic assumption as the first partition is expected to be well protected against transmission errors). For the current block, however, in order to decode its first partition, the receiver is required to provide the reference block represented by the same upper zone (i.e., $Z_{1, m}$ ) when it was decoded. Under these conditions, we can write the reconstructed reference block, after DCT conversion, with the same upper-zone as

$$
\begin{aligned}
& \underline{Z}_{1, m}\left\{\operatorname{DCT}\left(S_{f-1}\right)\right\} \\
& \quad=Z_{1, n}\left\{\operatorname{DCT}\left(S_{f-1}\right)\right\}+\underline{Z}_{n+1, m}\left\{\operatorname{DCT}\left(S_{f-1}\right)\right\}
\end{aligned}
$$

where $\underline{Z}_{n+1, m}$ represents a zone that is effected by errors. We assume that the receiver is designed to force these corrupted coefficients to zero. Subsequently, to decode the DCT coefficients covered by the first partition of the current block, the prediction error signal in (22) should be added to the reconstructed reference block with the same upper-zone, as shown in (27). Thus, from (22) and (27), the DCT coefficients of the first partition at the receiver may be expressed as

$$
\begin{aligned}
\underline{Z}_{1, m}\left\{\operatorname{DCT}\left(S_{f}\right)\right\}=Z_{1, m}\left\{\operatorname{DCT}\left(S_{f}-S_{f-1}\right)\right\} \\
\quad+Z_{1, n}\left\{\operatorname{DCT}\left(S_{f-1}\right)\right\}+\underline{Z}_{n+1, m}\left\{\operatorname{DCT}\left(S_{f-1}\right)\right\} .
\end{aligned}
$$

The absolute difference value between the reconstructed upperzone, with and without transmission errors, at the second partition represents the distortion value for the coding block. Thus, from (26) and (28), the following expression can be shown:

$$
\begin{aligned}
\left|Z_{1, m}\left\{\operatorname{DCT}\left(S_{f}\right)\right\}-\underline{Z}_{1, m}\left\{\operatorname{DCT}\left(S_{f}\right)\right\}\right| \\
\quad=\left|Z_{(n+1), m}\left\{\operatorname{DCT}\left(S_{f-1}\right)\right\}-\underline{Z}_{n+1, m}\left\{\operatorname{DCT}\left(S_{f-1}\right)\right\}\right| .
\end{aligned}
$$

The distortion term in (29) represents the amount of drift between the local decoder (24) and remote decoder (28) for the first partition of the current block. This indicates that the first partition has to rely on the channel condition in which the second partition is received. Its visual effects may depend on the number, as well as the magnitudes, of the nonzero coefficients that fall within the differential zone $Z_{m-n}$. Bear in mind that, in order to achieve a fixed-rate transmission for each partition, splitting of the VLC codewords between the two partitions is expected to change from time to time. As will be shown later, the progression of such distortion and its visual effects does not appear to be of serious concern-as far as the INTRA refresh mode can be accomplished in a reasonable interval. $^{2}$

Alternatively, the splitting of the DCT coefficients can be arranged before VLC coding. In this case the same number of lower frequency DCT coefficients (in a zigzag scanning order) is always selected for inclusion in the first partition (this region will be referred to as the upper-zone DCT). Although this approach has the advantage of providing SNR scalability, it can cause a substantial increase in bit rates. This is mainly due to the break up of the run-level symbol near the DCT cut-off region, which may increase the number of bits per block. In addition, for CBR transmission, this would require updating the quantization index for each partitioned layer by involving the encoder in the splitting process. In contrast to VLC-based partitioning, splitting can be accomplished entirely independently of the encoder as it will be required to operate at the entropy level.

\section{VLC-Based Partitioning}

Based on the ITU-T H.263 coding standard [9], we now present a VLC-based partitioning scheme suitable for transmission over our twin-class 16-QAM. It should be noted that the partitions are required to be of a fixed rate and often of

\footnotetext{
${ }^{2}$ To avoid the accumulation of inverse transform mismatch error, each MB is coded in INTRA mode at least once every 132 times when coefficients are transmitted for this MB in P-pictures.
} 
differing sizes in order to match to the transmission rates for C-1 + FEC1 and C-2 + FEC2 subchannels.

In our discussion so far, we have assumed that the first partition can always accommodate all the header hierarchy and, thus, splitting has to rely only on the VLC-coded DCT coefficients. In contrast, we observed that for video sequences with low motion activities, the header information far outweighs the VLC-coded coefficient data when the bit rate drops below certain levels. This indicates that a robust partitioning MB-header may also need to be transported via the second partitions (split-header). Furthermore, since we are dealing with CBR transmission, the cut-off level for splitting the bitstream needs to be updated in accordance with the relative buffer fullness of the individual channels. On this basis, the cut-off level is adjusted by the amount of data which needs to be transferred from one bitstream to another. This is shown by the adjustable indicator in Fig. 7. When the indicator moves toward the left, less data will be transported via the first bitstream and vice versa. To combine the two bitstreams at the receiver, the location of the adjustable indicator, which will be referred to as "cut-off value," should be included in the header of the particular transmission layer on which it is updated (e.g., picture-layer, GOB layer, MB layer).

Here, we have considered updating this information at the GOB level. Under this condition, a separate field is designated in the picture header (after PSC) and in every GOB header of the first bitstream for transmitting the cut-off value. To avoid any substantial increase in the overall bit rate, only a 2-bit header is allocated for transmitting and updating the cut-off level. In addition, to enhance the dynamic range of the adjustable indicator, the cut-off value is encoded differentially with reference to the previous position of the adjustable indicator (see Fig. 7). As illustrated in Fig. 8, five levels (L-0, L-1, L-2, L-3, and L-4) are used to signify the splitting region.

For example, suppose the splitting percentage of the first bitstream falls in the L-1 region; this means that significantly more data should be transported via the first partition in the next GOB. In this case, the amount of data that needs to be transferred to the first partition should go up by two steps from the previous position (e.g., moving the indictor in Fig. 7 toward the right by two steps).

If the splitting percentage falls in L-2, the splitting mechanism will move by only one step to the right. Likewise, when the splitting percentage falls within L-4 or L-3, the transfer of data will be from the first bitstream to the second. If percentage splitting falls in between L-0, the cut-off value will remain the same. Please note that the 2-bit cut-off value can be embedded into the $\mathrm{GN}^{3}$ if the input video format is QCIF or less [13].

1) Splitter: Now we discuss the manner in which the two partitions are formed at the bitstream splitter. For this purpose, let us consider a scenario where the first VLC,${ }^{4}$ as marked by the adjustable indicator in Fig. 7, is transported via the first partition for the current GOB in a P-frame. This is shown in greater detail in Fig. 9. As illustrated, the first partition begins with the GOB header followed by the MB header and the first VLC from each block in the transmitting order. This process will continue until

${ }^{3} \mathrm{GN}$ is a fixed codeword of 5 bits, identifying the GOB number in the frame. ${ }^{4}$ For INTRA blocks, INTRA-DC codeword will be considered instead.

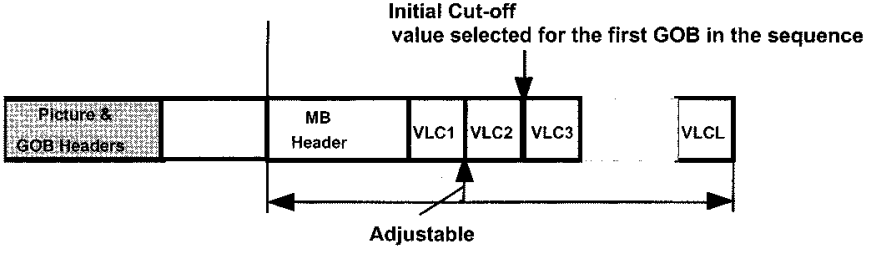

Fig. 7. Splitting mechanism within one GOB.

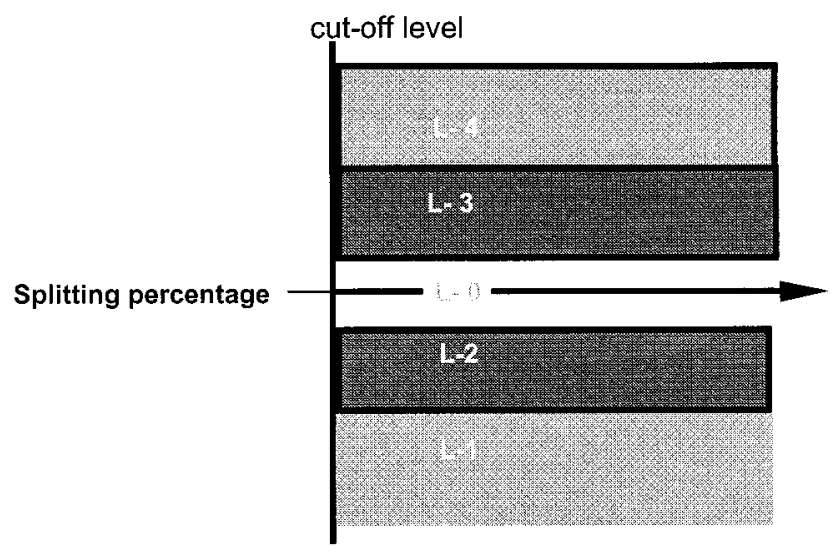

Fig. 8. Selection of the cut-off value using a 2-bit codeword.

the next GN in the upcoming GOB is detected. The remaining VLCs are subsequently transferred to the second partition in the same order. It should be noted that the second partition does not carry any VLCs from the blocks whose last VLCs are included in the first partition or identified as zero blocks by the MB header.

In order to resynchronize the two bitstreams at the receiver (i.e., pre-decoder), the second bitstream should be furnished with some header information. The header may consist of a picture start coded (PSC), and a temporal reference code (TR) at the picture layer and the GOB start code (GBSC), and the GN codeword at the GOB layer (i.e., H-2 in Fig. 9).

2) Pre-Decoder: At the receiver, the two bitstreams are joined together to form the original H.263 bitstream via a pre-decoder unit. The pre-decoder's initial task is to extract the cut-off value from the GOB header of the first bitstream. From the cut-off value, it can then determine which of the two bitstreams is carrying the MB information if the split-header is identified. Otherwise, the pre-decoder can decide from the cut-off value how many VLCs it should read from the first bitstream. If the last VLC is not included in the first bitstream, the predecoder will look for the remaining VLCs in the second bitstream. This process continues until the last VLC (last-run-level symbol) codeword (VLCL) is identified.

In our simulation model, when an error occurs on the second bitstream, the pre-decoder is able to detect it but unable to pinpoint its exact location. Under these conditions, the entire data on the second bitstream-which falls within one synchronization period-will be ignored. However, to reconstruct a decodable H.263 bitstream, all the blocks within a GOB which do not contain a VLCL (e.g., B1, B3, B4 in Fig. 9) in the first bitstream will be closed. This is done by replacing every last VLC (e.g., first VLC according to Fig. 9) by its equivalent VLCL codeword. If the cut-off value has identified a split-header, all the 

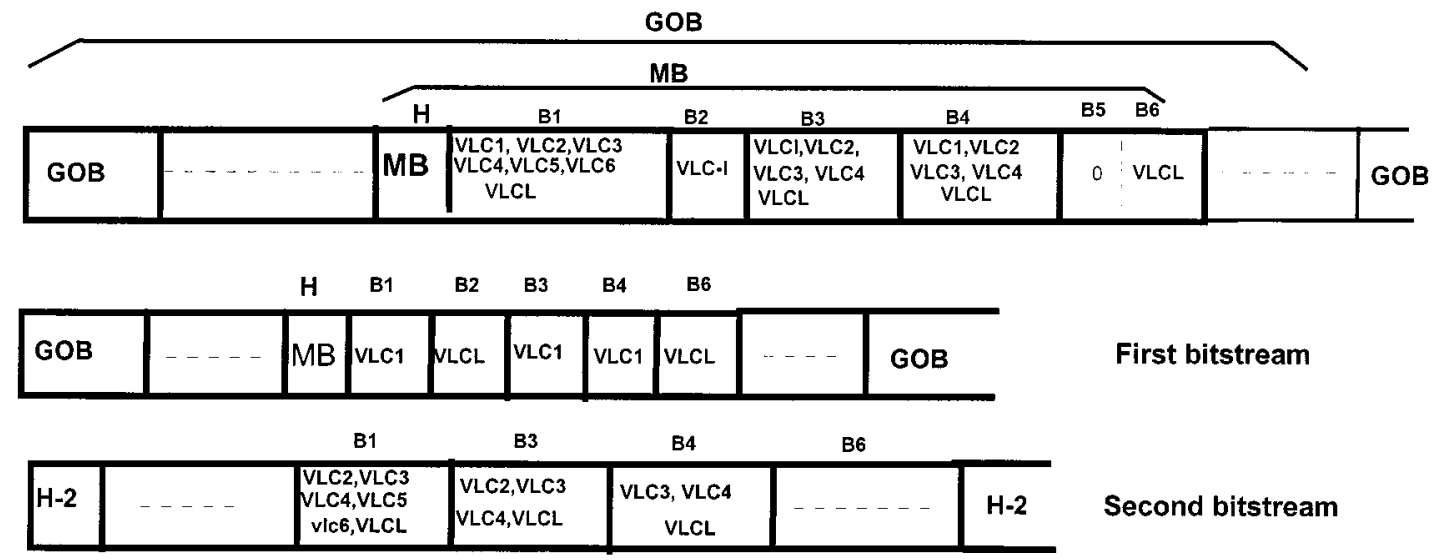

First bitstream

\section{Second bitstream}

Fig. 9. Two-layer VLC-based partitioning.

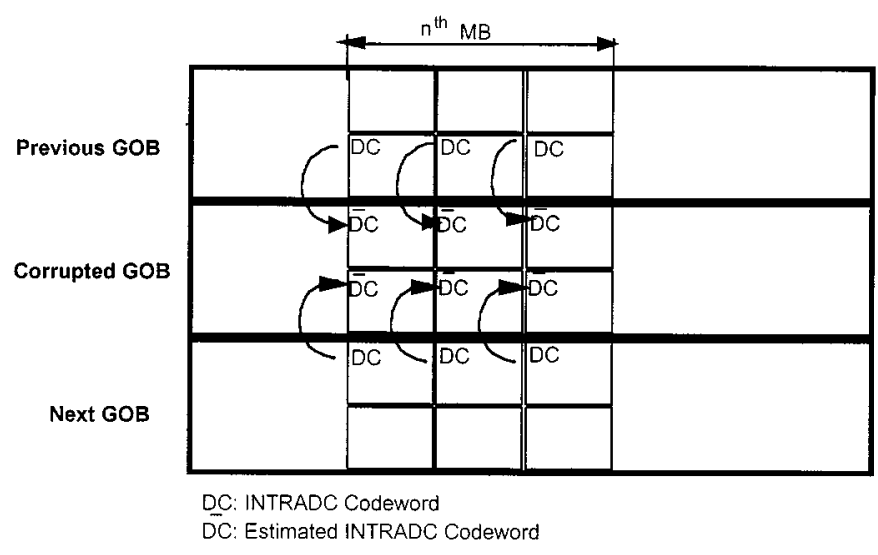

Fig. 10. Error-concealment procedure to estimate INTRADC codeword.

interframe blocks in the GOB will be considered not-coded by simply changing the COD flag.

For INTRA-pictures (I-pictures), however, the situation will be slightly different. ${ }^{5}$ For instance, to drop a corrupted second bitstream, there are two possible scenarios which would require special attention. The first scenario is when only the first codeword (INTRADC) is carried by the first bitstream. In this case, the predecoder will change the CBPY and CBPCM codes to signify that all the MB in this GOB do not carry any VLCs. The most unlikely scenario is when the INTRADC codeword is transported via the second bitstream. Under this condition, the predecoder selects an 8-bit codeword (preferably 00001111 ) for the blocks in the GOB. However, the effect of this causes the appearance of a gray ribbon covering the length of the I-picture with a width of one GOB (16 lines).

To conceal such a visual effect, each INTRADC codeword in the GOB will be replaced by the codeword from the neighboring GOB, as shown in Fig. 10. For the first and last GOB in the frame, all the INTRADC codewords will be replaced by the codewords from the previous or the proceeding GOBs, respectively. Please note that in our simulation model, if one of the two reference GOBs is corrupted by errors, the predecoder will use the other neighboring GOB is to estimate the INTRADC

\footnotetext{
${ }^{5}$ It should be noted that in the case of I-pictures it is not very likely to find GOBs with the split-header.
}

codeword. In the case where both reference GOBs are corrupted by errors, all the INTRASDC codewords will be replaced by 00001111.

As far as the first bitstream is concerned, a similar concealment approach has also been developed to realign the two bitstreams. However, its detailed description is rather tedious and beyond the scope of this paper.

\section{DISCUSSION AND RESULTS}

\section{A. Video Splitting}

Partitioning software programs were developed for the simulation. They performed the adaptive splitting as discussed in the previous section. In this program, the user defines the splitting percentage factor $X$ in order to set the transmission rates for the first and second bitstreams at $X \cdot R$ and $100-X . R$ respectively, where $X$ is the splitting percentage and $R$ is the overall transmission rate. The performance of the splitting scheme was then applied to the first 300 frames of two sequences known as "Salesman" and "Claire." Both sequences conformed to the QCIF format. Initially, the splitting algorithm was examined when these sequences were coded at $30 \mathrm{frames} / \mathrm{s}$ by considering equal sized partitioning (i.e., $X=50 \%$ ). In these experiments, except for the first frame which was intraframe coded, the remaining 290 frames were interframe coded. The results, in terms of the percentage difference between the two bitstreams coded at various fixed bit rates ranging from 16 to $128 \mathrm{kbits} / \mathrm{s}$, are plotted in Fig. 11. As can be observed, the 50\% splitting target has not been successfully met at bit rates below $32 \mathrm{kbits} / \mathrm{s}$. This is due to the fact that at very low bit rates, the number of VLCs tends to drop due to coarse quantization, leaving the second bitstream with insufficient information to transport. In fact, this is largely the result of utilizing full temporal resolution (30 frames/s), which should not be considered for video compression at very low bit rates.

In these experiments, we have observed that by lowering the coding frame rate to 10 frames/s, the partitioning can be successfully accomplished at a bit rate as low as $24 \mathrm{kbits} / \mathrm{s}$. In addition, as the bit rate goes up, the number of frames with split-header GOBs decreases. 


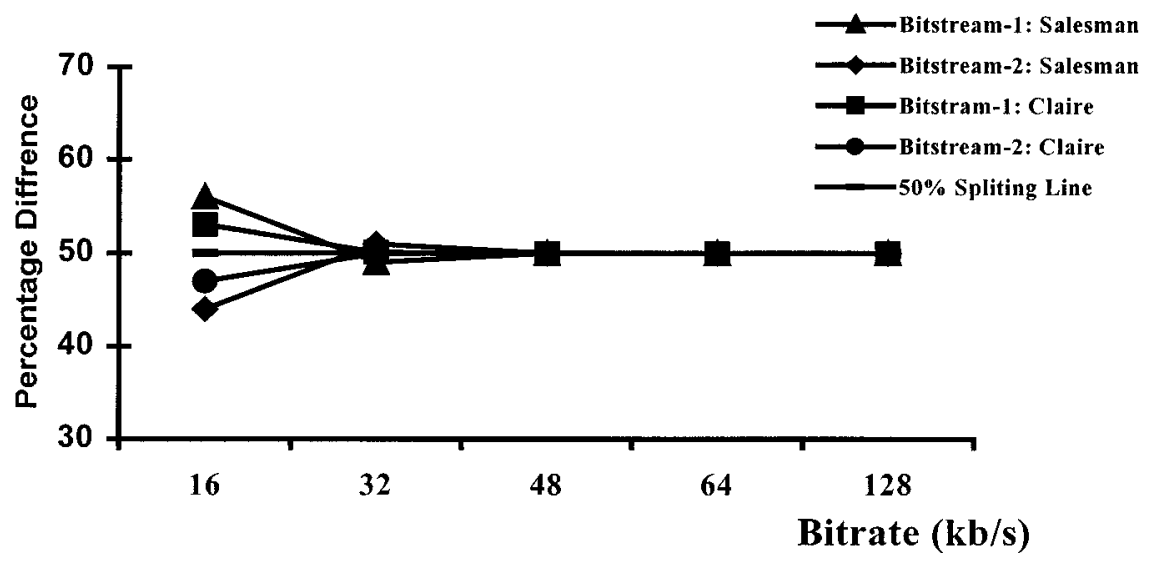

Fig. 11. Percentage difference between two bitstreams.

\section{B. Transmission}

The final stage of our experiments was concerned with the transmission aspects of the partitioned video signal. The end-to-end transmission system has been simulated using SPW simulation tools. As discussed in Section II, Gray-coded 16-QAM modulation with a baud rate of 24 was then used to transmit the pilot-assisted coded symbols. The modulated signal was then transmitted via AWGN channels under Raleigh fading (flat fading) conditions.

In these experiments, the ITU-T H.263-based video-compression technique was used to encode the input video signal. As before, two QCIF video sequences, "Salesman" and "Claire," were encoded at 10 frames/s. The coded bitstream was then partitioned into two bitstreams in such a way as to allow differing degrees of error protection for each partitioned bitstream. For FEC, convolutional codes were used to protect each bitstream independently. In addition, to minimize the effect of deep fades, a conventional block interleaver was applied following the channel encoding.

The pilot overheads were then inserted before multiplexing the bitstreams into a 4-bit symbol. As discussed earlier, a pilot symbol $\left(P_{\mathrm{C}-1} P_{\mathrm{C}-2}=\right.$ "1111") was first inserted after every 63 data symbols. In addition, a half symbol $\left(P_{\mathrm{C}-2}=\right.$ " 11 ") was also inserted on the second bitstream at every 8-symbol interval.

At the receiver, a QPSK demodulation was used to recover the first channel data $(\mathrm{C}-1)$. This was accomplished by achieving the primary channel estimation where a 2-bit pilot symbol $\left(P_{\mathrm{C}-1}=\right.$ "11") was first extracted by down sampling the received complex signal and then performing symbol-spaced interpolation for estimating the fading channel coefficients. These coefficients were then complex conjugated and multiplied to the received symbols before being sent to a QPSK slicer. A hard-decision Viterbi decoder had been used to decode the first bitstream.

The next step was the process of recovery of the second channel data; this was accomplished by first extracting a 2-bit data from the decoded first bitstream. This, together with the C-2 pilot symbol $\left(P_{\mathrm{C}-2}=\right.$ "11"), formed a full 4-bit pilot symbol at every frame (i.e., $T_{f}$ ). The pilot symbols were then used for the second-stage of channel estimation as shown in Figs. 3 and 4. In these experiments, the oversampling rate was 8 with a pulse shaping roll-off factor of .25 . The carrier frequency was $1.9 \mathrm{GHz}$.

In the first experiment, a $1 / 2$ rate convolution coding with a constraint length of 9 was applied to encode the first bitstream. The second bitstream was transmitted unprotected. For the first bitstream, the coding block structure consisted of 504 bits of data information and a 16-encoder tailor bit. This resulted in a total of 1024 encoded bits for the block (please note that the encoder tail bits were used to terminate the convolutional code). In addition, to minimize the effect of deep fades, a conventional block interleaver of $64 \times 16$ bits was then applied. Finally, by taking into account the overheads such as cut-off value, parity check bits, pilot information, and the second bitstream's synchronization bits (i.e., PSC and GBSC), the input video was encoded at $52.8 \mathrm{kbits} / \mathrm{s}$. In this case, the splitting percentage factor, $X$, was found to be $42 \%$.

At the receiver, both partitioned bitstreams were fed to the predecoder block. As mentioned earlier, the function of the predecoder is to re-synchronize, re-align, and finally perform a limited error concealment. Its performance largely depends on the size of its input buffers for storing the partitioned bitstreams for the re-alignment process. To avoid excessive delay, we have deployed a 2.0-kbit buffer for each channel. In these experiments, we observed that the effect of error bursts on the header information (e.g., PSC, TR, etc.) may extend well beyond a single P-frame. Consequently, this could seriously upset the resynchronization process where the next picture header may not be identified within the stored data. In this situation, the predecoder replaces the entire data in each corrupted frame by the successfully re-aligned data from the previous frame (freeze-frame).

However, under less severe fading conditions, the effect of error bursts is likely to be contained only within a few neighboring GOBs. In this case, upon detecting errors in the first channel, the entire data in any of the erroneously detected GOBs is forced to zero, resulting in a replacement of the reconstructed GOB from the reference frame. In this situation, all the MBs in the GOB are considered as uncoded (i.e., COD $=1$ ). In a case where the errors effect an I-frame, a new GOB is generated that will include only an INTRADC codeword for every block. The codeword is regenerated by interpolating the INTRADC values from the nearest blocks of the neighboring GOBs (see Fig. 10). For further details of the effect of transmission errors on the bit- 


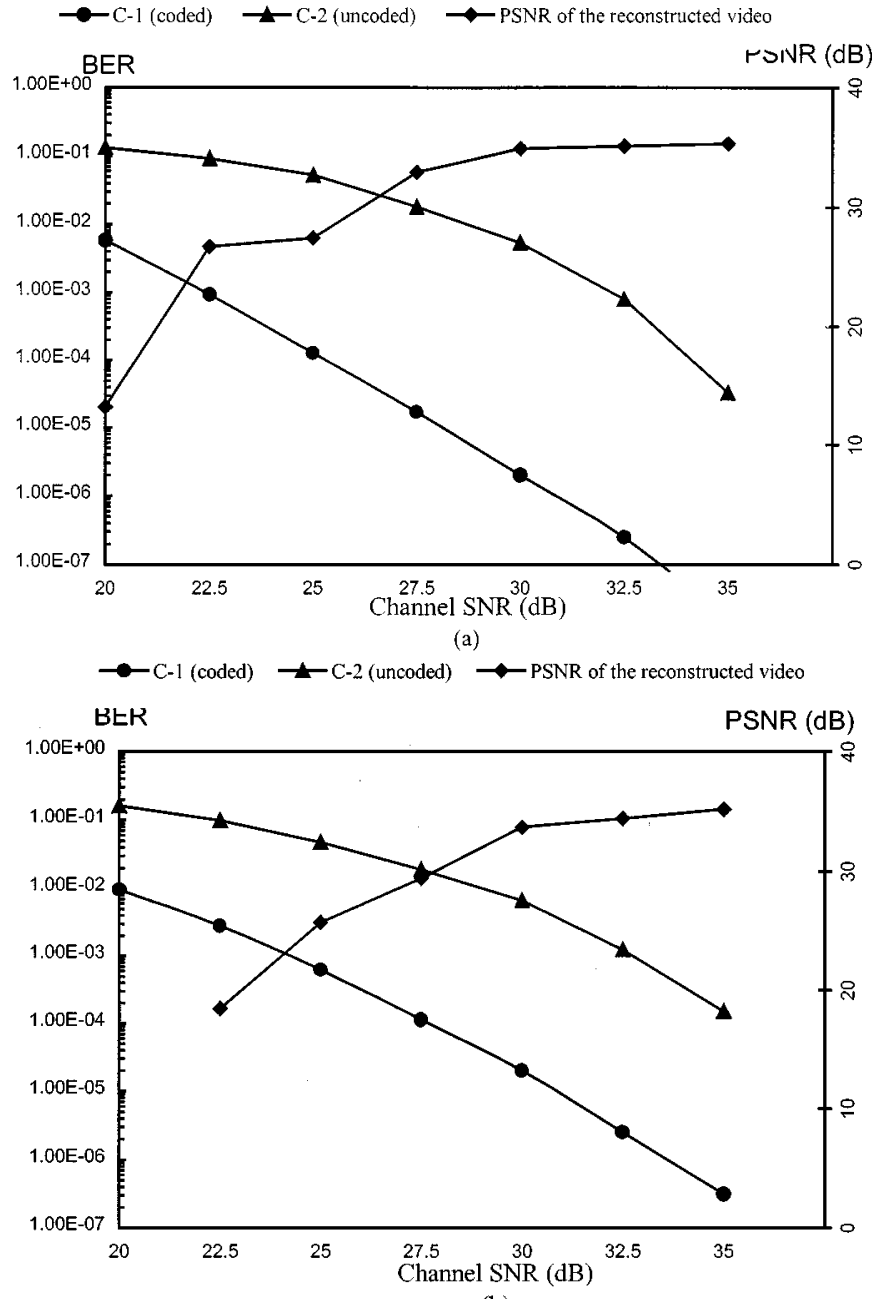

(b)

Fig. 12. Average PSNR of the reconstructed frames of the "Claire sequence" when transmitted via a twin-class 16-QAM. (a) $f_{D} T_{s}=0.01$. (b) $f_{D} T_{s}=$ 0.015 .

stream and the alignment process, please refer to Section V-B. Finally, the aligned bitstream is then forwarded to the standard ITU-T H.263 decoder.

Fig. 12 shows the average PSNR (peak-to-peak signal to rms noise ratio) of the reconstructed received video frames versus channel SNR $\left(E_{s} / N_{0}\right)$ for the "Claire" sequence. This figure also includes the BER results for $\mathrm{C}-1$ and $\mathrm{C}-2$. These results were obtained for a normalized Doppler frequency $\left(F_{d} T_{s}\right)$ of 0.01 [Fig. 12(a)] and 0.015 [Fig. 12(b)]. Furthermore, to generate a long string of binary data, input video sequences each consisting of 396 frames (30 frames/s), was repeated ten times and then coded at 10 frames/s. After the last frame of the first sequence was encoded the first frame of the repeated sequence was then encoded as I-frame, thus resulting in the I-frame reset period of 134 frames.

The results in Fig. 12 indicate that at lower $E_{s} / N_{0}$ values, the predecoder is unable to resynchronize the received bitstreams due to excessive noise. This situation could result in a severely distorted first frame (I-frame), followed by repeated freeze-frames. As for the higher channel SNR, the performance of the predecoder tends to improve considerably (e.g., depending on the mobile velocities). Such an improvement becomes more noticeable as the number of freeze-frames drops. In particular, it should be noted that such an improvement is closely related to the BER performance of the multipath resistant channel despite the relatively poor BER performance of the second channel.

For further justification, the second experiment was carried out with the objective of having identical BERs for both channels. This was mainly to evaluate the advantage of an unequal error protection strategy for transmission of the partitioned video bitstreams. In these experiments, secondary fade estimation was turned off. Instead, a conventional channel estimation, where a 4-bit pilot symbol is transmitted at the beginning of every frame of 19 symbols (i.e., $T_{f}=20$ ), was deployed. In this arrangement, the first channel data was transmitted unprotected, whereas the second channel data was encoded using a rate-compatible punctured convolutional code (RCPC) [14] at a rate of $2 / 5$. This code consisted of a $1 / 3$ rate convolutional code with the constraint length of 5 and the puncturing rate of 24/20 (4 symbols are deleted out of every 24 coded symbols). The coding was performed on a block of 476 bits of the second bitstream, resulting in a total of 1200 encoded symbols for every block (please note that a block interleaver of $60 \times 20$ bits has been applied). Taking into consideration all the overheads, the input video could then be encoded at the same bit rate of $52.8 \mathrm{kbits} / \mathrm{s}$, but with a splitting percentage factor of $72 \%$. Under these conditions, we observed that nearly the same BERs for both channels can be accomplished at $E_{s} / N_{0}=28 \mathrm{~dB}$ for the normalized Doppler frequency of $F_{d} T_{s}=0.01$. The results are shown in Table I. We have also included the results of the first experiments obtained under the same transmission environments.

Furthermore, for the sake of comparison, the transmission of a nonpartitioned H.263-coded bitstream was also conducted. In this case the RCPC rate was $4 / 5$ and this provided almost the same FEC overhead as in the other experiments in Table I. For this, the predecoder was designed in such a way that whenever errors are detected in any of the GOBs, the entire MBs in the GOB are considered to be uncoded. The results of this experiment under the same test environments are also shown in Table I.

These results indicate a far superior performance of the partitioned video bistream when transmitted over a twin-class QAM with unequal BER for each of its channels compared with the other two systems. To verify this subjectively, we compared the quality of the reconstructed video for all three systems of Table I. In these evaluations, we carried out each experiment using a repeated video sequences five times and each time reinitialized the noise generator differently. We then observed that the overall quality of the partitioned video transmitted with an equal BER (system 2), in terms of the number of freeze-frames, was slightly better than system 3 .

This clearly indicates the effectiveness of the video partitioning even without utilizing unequal error transmission. However, with a combination of the unequal error-protection scheme and the secondary fade estimation, we observed that quality of the reconstructed video is far better than the other two systems. Indeed, when the same experiments are repeated with higher channel SNR (e.g., BER $\mathrm{B}_{\mathrm{C}-1}<10^{-5}$ and $\mathrm{BER}_{\mathrm{C}-2}<10^{-3}$ ), the 
TABLE I

PERFormance COMPARISON BETWEen PARTITIONED AND NON-PARTITIONEd Transmission OF THE "SALESMAN" SEQUENCE

\begin{tabular}{l|c|l|l|l|l|l|l|l|l}
\hline System & $\begin{array}{c}\text { Video Bitrate } \\
(\mathrm{kb} / \mathrm{s})\end{array}$ & $\begin{array}{c}\text { Bit } \\
\text { Stream }\end{array}$ & $\begin{array}{l}\text { FEC- } \\
\text { rate }\end{array}$ & $\begin{array}{c}\text { Channel } \\
\text { estimation }\end{array}$ & $\begin{array}{c}\text { Pilot } \\
\text { Frame }\end{array}$ & $\mathrm{F}_{\mathrm{d}} \mathrm{T}_{\mathrm{s}}$ & $\begin{array}{l}\mathrm{E}_{\mathrm{s} / \mathrm{N}_{0}} \\
(\mathrm{~dB})\end{array}$ & \multicolumn{1}{c}{$\mathrm{BER}$} & $\begin{array}{l}\text { PSNR } \\
(\mathrm{dB})\end{array}$ \\
\hline Partitioned & 52.8 & 2 & $\begin{array}{l}1 / 2 \\
-\end{array}$ & $\begin{array}{l}\text { Two- } \\
\text { stage }\end{array}$ & $\begin{array}{c}64 \\
8\end{array}$ & 0.01 & 28 & $\begin{array}{l}1.2 \times 10^{-4} \\
0.011\end{array}$ & 32.8 \\
\hline Partitioned & 52.8 & 1 & $\begin{array}{l}\text { Non } \\
2 / 5\end{array}$ & $\begin{array}{l}\text { One- } \\
\text { stage }\end{array}$ & 20 & 0.01 & 28 & $9.1 \times 10^{-4}$ & 25.7 \\
\hline $\begin{array}{l}\text { Non- } \\
\text { Partitioned }\end{array}$ & 50.944 & - & $4 / 5$ & $\begin{array}{l}\text { One- } \\
\text { stage }\end{array}$ & 20 & 0.01 & 28 & $8.3 \times 10^{-4}$ & \\
\hline
\end{tabular}

quality of the system 1 becomes almost indistinguishable with the quality of the transmitted video signal. Finally, we should emphasize that increasing the size of the predecoder buffers can considerably reduce the number freeze-frames in the received bitstreams mainly due to better detection of the uncorrupted synchronization information. Another important factor is utilizing more frequent INTRA-refresh option at the encoder, but this would be at the expense of the increasing the overall bit rate.

\section{CONCLUSION}

The main objective in this paper was to investigate the transmission of compressed video bitstreams over multipath fading environments via a twin class 16-QAM modulation system. This required developing a robust video partitioning scheme where each partitioned bitstream can be transmitted by a separate 16-QAM transmission channel. It is well known that the BER performance of the two LSBs (referred to as the second channel) of the 4 bits/symbol of a Gray-coded 16-QM tends to deteriorate rapidly as the Doppler frequency increases. We have shown that such deterioration can be reduced considerably if more frequent pilot information is transmitted for the second channel. A second stage of fade estimation is then proposed which can significantly improve the second channel performance.

Subsequently, we have discussed two methods of partitioning which are based on separating the DCT data before and after VLC coding. It was shown that while the former can be more suitable for SNR scalability, the latter was considered for our application mainly due to its partitioning efficiency. The partitioning method was then applied to split the compressed video bitstream. The ITU-T H.263 coding standard was used to encode the QCIF video input.

Finally, the end-to-end transmission of the partitioned video bitstreams using the above 16-QAM system was evaluated under various transmission environments. We have shown that a combination of the twin class 16-QAM with the secondary fade estimation and the video partitioning forms an effective method in transmitting video information over mobile channels.

\section{APPENDIX}

\section{DERIVATION OF THE ERROR PROBABILITY OF THE SECOND CHANNEL 16-QAM}

To obtain the average BER of the second channel, we assume that the decision boundaries for the LSB of the $I$ and $Q$ components should be adjusted by an average fade factor of
$E\{r\}=r_{0}$. For example, let us first consider that a logical zero is transmitted for an $I$ or $Q$ component. In this case, the distance of a constellation point from the boundary of its decision region would be $d=\left(2 r_{0}-r\right) a$ instead of $d=(2 a-a)=a$ in a nonfading case. Under this condition, the second channel average bit-error probability for the logical zero using (4) can be shown as

$$
p_{\mathrm{c}-2}^{0}=\int_{0}^{\infty} \frac{1}{\gamma_{0}} e^{-\left(\gamma_{i} / \gamma_{0}\right)} Q\left\{\left(\frac{2 r_{0}}{r}-1\right) \sqrt{\frac{\gamma_{i}}{5}}\right\} d \gamma_{i} .
$$

In the case when the logical one is transmitted, the protection distance would be $d=\left(3 r-2 r_{0}\right) a$ and therefore the average bit-error probability is

$$
p_{\mathrm{c}-2}^{1}=\int_{0}^{\infty} \frac{1}{\gamma_{0}} e^{-\left(\gamma_{i} / \gamma_{0}\right)} Q\left\{\left(\frac{3 r_{0}}{r}-2\right) \sqrt{\frac{\gamma_{i}}{5}}\right\} d \gamma_{i} .
$$

For a Rayleigh model, the ratio $r_{0} / r$ can be shown as

$$
\frac{r_{0}}{r}=\frac{1}{2} \sqrt{\frac{\pi \gamma_{0}}{\gamma_{i}}}
$$

Replacing the above ratio in (A1) and (A2) would result in

$$
\begin{aligned}
& p_{\mathrm{c}-2}^{0}=\int_{0}^{\infty} \frac{1}{\gamma_{0}} e^{-\left(\gamma_{i} / \gamma_{0}\right)} Q\left\{\frac{\sqrt{\pi \gamma_{0}}-\sqrt{\gamma_{i}}}{\sqrt{5}}\right\} d \gamma_{i} \\
& p_{\mathrm{c}-2}^{1}=\int_{0}^{\infty} \frac{1}{\gamma_{0}} e^{-\left(\gamma_{i} / \gamma_{0}\right)} Q\left\{\frac{3 \sqrt{\pi \gamma_{0}}-4 \sqrt{\gamma_{i}}}{\sqrt{20}}\right\} d \gamma_{i} .
\end{aligned}
$$

Simplifying (A4) and (A5), the following equations are obtained:

$$
\begin{aligned}
& p_{\mathrm{c}-2}^{0}=\frac{1}{2} e^{\frac{-\pi \gamma_{0}}{10}}\left[1+\sqrt{\frac{\gamma_{0}}{10+\gamma_{0}}} e^{\frac{\pi \gamma_{0}}{10\left(\gamma_{0}+10\right)}}\right] . \\
& p_{\mathrm{c}-2}^{0}=\frac{1}{2} e^{\frac{-9 \pi \gamma_{0}}{40}}\left[1+\sqrt{\frac{4 \gamma_{0}}{4 \gamma_{0}+10}} e^{\frac{9 \pi \gamma_{0}^{2}}{40 \gamma_{0}+100}}\right] .
\end{aligned}
$$

Thus, the total bit-error probability for channel 2 under Rayleigh fading conditions is

$$
\overline{p_{\mathrm{c}-2}}=\frac{1}{2}\left(p_{\mathrm{c}-2}^{0}+p_{\mathrm{c}-2}^{1}\right)
$$

or

$$
\begin{aligned}
\overline{P_{\mathrm{c}-2}}= & \frac{1}{4} e^{\frac{-\pi \gamma_{0}}{10}}\left[1+\sqrt{\frac{\gamma_{0}}{10+\gamma_{0}}} e^{\frac{\pi \gamma_{0}^{2}}{10\left(\gamma_{0}+10\right)}}\right] \\
& +\frac{1}{4} e^{\frac{-9 \pi \gamma_{0}}{40}}\left[1+\sqrt{\frac{4 \gamma_{0}}{4 \gamma_{0}+10}} e^{\frac{9 \pi \gamma_{0}^{2}}{40 \gamma_{0}+100}}\right] .
\end{aligned}
$$




\section{ACKNOWLEDGMENT}

The author thanks D. Ainsworth, V. Ong, and F. Chen for developing some of the software programs used in this investigation. He also thanks Dr. L Miller and Dr. K. Ban for their useful comments.

\section{REFERENCES}

[1] R. Stedman, H. Gharavi, L. Hanzo, and R. Steele, "Transmission of subband coded images via mobile channels," IEEE Trans. Circuits Syst. Video Technol., vol. 3, pp. 15-26, Feb. 1993.

[2] W. T. Webb and L. Hanzo, Modern Quadrature Amplitude Modulation: Principles and Applications for Fixed and Wireless Channels. New York: IEEE Press/Pentech, 1994, p. 557.

[3] J. K. Cavers, "An analysis of pilot symbol assisted modulation for Rayleigh fading channels," IEEE Trans. Veh. Technol., vol. 40, pp. 689-693, Nov. 1991.

[4] S. Sampei and T. Sunaga, "Rayleigh fading compensation for QAM in land mobile radio communications," IEEE Trans. Veh. Technol., vol. 42, pp. 137-146, May 1993.

[5] W. C. Lee, Mobile Communications Engineering. New York: McGraw-Hill, 1985.

[6] W. C. Jakes, "A comparison of specific space diversity technique for reduction of fast fading in UHF mobile radio systems," IEEE Trans. Veh. Technol., vol. VT-20, pp. 81-93, Nov. 1971.

[7] S. Stein, "Fading channel issues in system engineering," IEEE J. Select. Areas Commun., vol. SAC-5, Feb. 1987.

[8] Video codec for audio visual services at $p \times 64 \mathrm{kbit} / \mathrm{s}$, ITU-T H.261 Recommendation, Mar. 1993.

[9] Video coding for low bitrate communication, ITU-T Recommendation H.263, Feb. 1998

[10] Video coding for low bitrate communication, ITU-T Recommendation H.263, Version 2, Jan. 1998.

[11] MPEG-1 Int. Std. Coding of Moving Pictures and Assisted Audio for Digital Storage Media up to About 1.5 Mbit/s, Video Recommendation ITU-T H.262, ISO/IEC 13 818-2, Jan. 1995.

[12] MPEG-2 Int. Std., Video Recommendation ITU-T H.262, ISO/IEC 13 818-2, Jan. 1995
[13] H. Gharavi and S. M. Alamouti, "Multi priority video transmission for third generation wireless communication systems," Proc. IEEE, vol. 87, pp. 1751-1763, Oct. 1999.

[14] J. Hagenhauer, "Rate-compatible punctured convolutional codes (RCPC codes) and their applications," IEEE Trans. Commun., vol. 36, Apr. 1988.

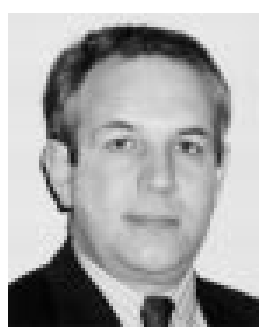

Hamid Gharavi (F'92) received the Ph.D. degree from Loughborough University of Technology, Loughborough, U.K., in 1980

He joined AT\&T Bell Laboratories, Holmdel, in 1982. He was then transferred to Bell Communications Research (Bellcore) after the AT\&T-Bell divestiture, where he became a Consultant on video technology and a Distinguished Member of Research Staff. In 1993, he joined Loughborough University as Professor and Head of Communication Engineering. Since September, 1998, he has been with the National Institute of Standards and Technology (NIST), Gaithersburg, MD. He was a core member of the Study Group XV (Specialist Group on Coding for Visual Telephony) of the International Communications Standardization Body CCITT (ITU-T). He was selected as one of the six university academics to be appointed to the U.K. Government's Technology Foresight Panel in Communications to consider the future through 2015 and make recommendations for allocation of key research funds. He served as a Member of the Communication Sector Panel of the U.K. Technology Foresight Program, Cabinet Office, Office of Science and Technology, during 1994-1995. His research interests include video/image transmission, wireless multimedia, mobile communications, and third generation wireless systems. He holds eight U.S. patents related to these topics.

Dr. Gharavi has been an Associate Editor of the IEEE TRANSACTIONS ON CIRCUITS AND SYSTEMS FOR VIDEO TECHNOLOGY since 1996 and has been a Guest Editor for a number of special issues, the most recent being Wireless Multimedia Communications for the PROCEEDINGS OF THE IEEE (October 1999). He was an Associate Editor of IEEE TRANSACTIONS ON CIRCUITS AND SYSTEMS (1987-1989) and served as Chairman of the VLSI Systems and Applications Committee of the IEEE Circuits and Systems (CAS) Society (1989-1991). He received the Charles Babbage Premium Award of the Institute of Electronics and Radio Engineering in 1986 and the IEEE CAS Society Darlington Best Paper Award in 1989. 